\title{
Emerging technologies illuminate facets of photosynthesis in cyanobacteria
}

\author{
Devaki Bhaya $^{1} \cdot$ Peter Lindblad $^{2}$
}

Published online: 22 September 2015

(C) Springer Science+Business Media Dordrecht 2015

It is with great pleasure that we present a special volume of Photosynthesis Research entitled Emerging technologies illuminate facets of photosynthesis in cyanobacteria which includes eleven invited reviews and original papers.

When we invited authors to contribute to this special volume our reasoning and rationale was that several new research groups were interested in the possibilities, usefulness, and power of cyanobacteria in biotechnological applications (see recent reviews by Xue and He 2015; Angermayr et al. 2015, Oliver and Atsumi 2014). Thus, a volume reviewing some of the new approaches being developed for use in cyanobacteria would find wide readership. In addition, it would give students and researchers a sense of excitement about powerful technologies that are beginning to make an impact on cyanobacterial research. Some of these technologies were initially developed for other model systems but optimizing them for cyanobacteria represents a most important step forward.

Photosynthesis is central and unique in photoautotrophs affecting, directly or indirectly, almost all aspects of cyanobacterial biology. In this special issue, different aspects and topics of cyanobacterial photosynthesis such as the light harvesting machinery and reaction centers, carbon fixation and metabolism, and evolution are reviewed and

Devaki Bhaya

dbhaya@carnegiescience.edu;

http://dpb.carnegiescience.edu/labs/bhaya-lab

Peter Lindblad

Peter.Lindblad@kemi.uu.se

Carnegie Institution for Science, Stanford, CA 94305, USA

2 Microbial chemistry, Department Chemistry - Angstrom, Faculty of Science and Technology, Uppsala University, Box 523, 75120 Uppsala, Sweden recent progress is highlighted. We also touch on other emerging areas of interest relevant to the development of cyanobacteria as an industrial usable chassis. Our initial list covered a very diverse list of potential contributors, but obviously not everyone could accept our invitation.

In more detail, the special issue on Emerging technologies illuminate facets of photosynthesis in cyanobacteria contains contributions on the evolution and diversification of cyanobacterial photochemical reaction centers (Cardona), ${ }^{13} \mathrm{C}$ flux analysis of cyanobacterial metabolism (Adebiyi, Jazmin and Young), proteomic approaches to examine cyanobacterial photosynthesis (Battchikova, Angeleri and Aro), and an update on the auxiliary genes carried by viruses that affect light and dark reactions of photosynthesis (Puxty, Millard, Evans and Scanlan). Additional papers address the energy arriving at the photochemical reaction centers with a focus on the orange carotenoid protein-related photoprotection and state transitions (Kirilovsky). Kerfeld and co-workers describe the bioinformatic analysis of the distribution of inorganic carbon transporters and suggest targets for bioengineering to increase $\mathrm{Ci}$ uptake by cyanobacteria (Guadana, Zarzycki, Moparthi and Kerfeld), and Larom et al. examine the effect of a Photosystem II D1-K238E mutation on the electrical current production by cyanobacterial thylakoid membranes in a bio-photoelectrochemical cell (Larom, Kallman, Sape, Pinhassi, Rothschild, Dotan, Ankonina, Schuster and Adir). Finally, we have included four articles that cover a diversity of molecular tools/approaches that are relevant to cyanobacteria which touch on some of specific and unusual features of cyanobacteria and the challenges of working with them. These include articles on the live-cell imaging of cyanobacteria (Yokoo, Hood and Savage), the challenges of metagenomics and singlecell genomics approaches for exploring cyanobacterial 
diversity (Davison, Hall, Zare and Bhaya), the roles of translation initiation factor 3 families in regulating cyanobacterial and chloroplast gene expression (Nesbit, Whippo, Hangarter and Kehoe), and the genetic and genomic analysis of RNases in model cyanobacteria (Cameron, Gordon and Pfleger).

It is our aim and hope that this special issue on Emerging technologies illuminate facets of photosynthesis in cyanobacteria with a collection of eleven up-to-date papers written by internationally leading scientists will inspire both younger and established researchers as well as students at all levels to further explore the fundamental knowledge of cyanobacterial biology as well as the development of cyanobacteria as photosynthetic microbial chassis for biotechnological applications (see recent reviews by Xue and $\mathrm{He}$ 2015, Angermayr et al. 2015, Oliver and Atsumi 2014). We would also like to alert readers to the special volume edited by Mayfield and
Golden "Biofuels and Bio-products from Photosynthetic Microorganisms" that appeared in Photosynthesis Research, March 2015.

Devaki Bhaya

Peter Lindblad

\section{References}

Angermayr SA, Gorchs Rovira A, Hellingwerf KJ (2015) Metabolic engineering of cyanobacteria for the synthesis of commodity products. Trends Biotechnol 33(6):352-361

Oliver JWK, Atsumi S (2014) Metabolic design for cyanobacterial chemical synthesis. Photosynth Res 120(3):249-261

Xue Y, He Q (2015) Cyanobacteria as cell factories to produce plant secondary metabolites. Front Bioeng Biotechnol 28(3):57 